\title{
Phase Space Description of Nonlinear Directional Couplers
}

\author{
David Artigas and Federico Dios
}

\begin{abstract}
A comprehensive analysis of the symmetric nonlinear directional coupler as a function of the excitation conditions is reported. The analysis is based on a coupled-mode technique in normalized (dimensionless) parameters, using the linear supermodes of the guiding structure, most of the results being derived from the Hamiltonian of the resulting nonlinear equations. The exchange of power between the branches in the coupler is graphically described by means of the trajectories of the motion in the phase space. The effect of saturation of the nonlinear index has been included.
\end{abstract}

\section{INTRODUCTION}

I T RECENT years great interest has been shown in using nonlinear waveguided devices within the fields of optical communication technology and ultrahigh-speed data processing [1]. The main idea consists of controlling light with light, taking advantage of the nonlinear optical effects without the necessity of including optoelectronic conversion processes. Among integrated optical devices, the nonlinear directional coupler (NLDC), where the media show a Kerr-like nonlinearity has received the most attention. The first analytical model making use of the coupled-mode theory was proposed by Jensen [2]. This work was followed by other theoretical models, which improved on the initial one. First, there were those who tried to improve the coupled-mode theory by taking the modes of the five-layer structure [3], including the nonlinear coupling effect and $N$-effect conditions [4], or using the nonlinear modes of each isolate waveguide [5] or of the five-layer structure [6]. More realistic factors, which affect the coupler features, were considered in other works, that is, saturation of the nonlinear index [7] and losses [8]-[9], diffusion length for a nonlocal nonlinearity [10], coupler asymmetry [11], and temporal field evolution [12]. Other works dealt with the stability problem [13], [14]. The NLDC was also studied numerically using the beam propagation method [15], [16], which gave more accurate numerical results, and experimentally [17]-[19], where the results were compared with theory.

Recently, additional comprehensive NLDC analysis has been reported. Snyder et al. [20] developed graphical power flow representations valid in a wide range of non-

Manuscript received May 17, 1993; accepted Oct. 18, 1993. The work of D. Artigas was supported by a grant from the D.G.R. of the Catalunya Government.

The authors are with the Department of Signal Theory and Communications, Universitat Politècnica de Catalunya, 08080-Barcelona, Spain. IEEE Log Number 9401168. linear couplers with nonlinearity laws other than the classical Kerr-like one. Phase space portraits using Stokes parameters [13], [21] and potential-well models [22] have also been used in order to give an intuitive view of NLDC features. Our work fits into this group. In our case, we use the Hamiltonian associated with the coupled-mode equations where normalized dimensionless parameters have been included in order to reduce the number of variables and to obtain universal curves of the device. It allows us to obtain closed expressions as well as qualitative and illustrative plots of the trajectories of the motion in the phase space, which give valuable information on its evolution, behavior, and stability in terms of the excitation conditions and of the normalized geometrical parameters.

This paper is organized as follows. In Section II we find the scaling rules of our problem, the coupled-mode equations and the Hamiltonian. Section III is devoted to describing the NLDC with Kerr-like media by means of the Hamiltonian and phase space portraits. In Section IV we show the results for non-Kerr-like media. The stability is discussed in Section V, followed by our conclusions.

\section{Coupled-Mode Equations with Normalized PARAMETERS}

We will focus our study on the NLDC with a symmetric structure, as depicted in Fig. 1. Our device consists of a layer with width $2 w$ and linear refractive index $n_{3}$ sandwiched between two guides of thickness $d$ having the same index $n_{2}$. The cladding and substrate linear refractive index is $n_{1}$. Moreover, we will study only the case $n_{1} \leq n_{3}$ $<n_{2}$. All the media have a non-Kerr-like nonlinear intensity-dependency given by

$$
n^{2}=n_{i}^{2}+\alpha_{k}|E|^{2}-\beta_{k}|E|^{4}
$$

with $i=1,2,3$ for each medium, where the second term in the series expansion of the nonlinear refractive index has been considered in order to simulate the saturation [23]. Loss-free media are also assumed.

In our work we follow the formalism proposed by Silberberg and Stegeman [3]. A comparison of this approach with other works can be found in [12]. Taking the symmetric and the antisymmetric TE lowest-order modes of the five-layer linear structure, where the nonlinear effect appears in the variations of the complex amplitudes $A_{i}(z)$, 


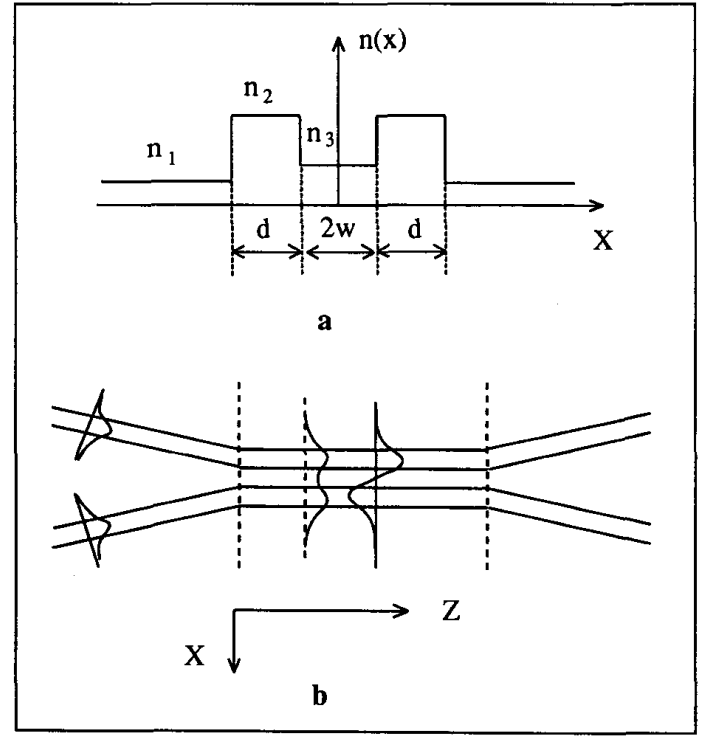

Fig. 1. The symmetric nonlinear directional coupler studied in this work.

the electric field can be written as

$$
\vec{E}(x, z)=\left[A_{o}(z) E_{0}(x) e^{-j \beta_{0} z}+A_{1}(z) E_{1}(x) e^{-j \beta_{1} z}\right] \vec{y}
$$

$E_{0}(x) e^{-j \beta_{0} z}$ being the $\mathrm{TE}_{0}$ (symmetric) mode and $E_{1}(x) e^{-j \beta_{12}}$ the $\mathrm{TE}_{1}$ (antisymmetric) mode, where $z$ is the propagation direction and $x$ the transverse axis.

The study of NLDC behavior requires the determination of the allowed values of the propagation constant $\beta$ and the field $E_{i}$ of the two first linear modes (in our case) as a function of the waveguide parameters $n_{1}, n_{2}, n_{3}, d$, $w$ and the free-space wavelength $\lambda$. These can be reduced by introducing appropriate normalized parameters and scaling rules for the five-layer waveguide [24]. In a way similar to the work of Recolons et al. [25], we define the following normalized parameters:

$$
\begin{aligned}
b_{i} & \equiv \frac{N_{i}^{2}-n_{1}^{2}}{n_{2}^{2}-n_{1}^{2}} \\
a & \equiv \frac{n_{3}^{2}-n_{1}^{2}}{n_{2}^{2}-n_{1}^{2}} \\
V_{1} & \equiv k_{o} d\left(n_{2}^{2}-n_{1}^{2}\right)^{1 / 2} \\
V_{2} & \equiv w / d
\end{aligned}
$$

$b_{i}$ being the normalized effective index for the eigenmodes with $N_{i}=\beta_{i} / k_{o}$ and $k_{o}=2 \pi / \lambda, a$ the asymmetry coefficient, which must be understood as a relation between the refractive indexes $n_{1}$ and $n_{3}, V_{1}$ the normalized frequency for the guiding layer, and $V_{2}$ the normalized separation between the guiding layers. We also write the normalized modal field in the form

$$
\Psi_{i}(\mathfrak{X}) \equiv \frac{E_{i}(\mathfrak{X})}{E_{c}}
$$

$E_{c}$ being a constant that will be specified later on and $X$ $=x / d$ the transverse dimensionless coordinate. The value of the effective index $b_{i}$ is given by the dispersion equations written in terms of the other three parameters in a similar way as in [24]. This can be obtained from the continuity of the tangential electric and magnetic normalized modal fields in the refractive index profile discontinuities as

$$
\begin{aligned}
\tan ^{-1} & \sqrt{\frac{b_{i}}{1-b_{i}}} \\
& +\tan ^{-1}\left[\sqrt{\frac{b_{i}-a}{1-b_{i}}}\left[\tanh \left(V_{2} V_{1} \sqrt{b_{i}-a}\right)\right]^{(-1)^{i}}\right] \\
& -V_{1} \sqrt{1-b_{i}}=0
\end{aligned}
$$

where $i=0$ is the $\mathrm{TE}_{0}$ mode and $i=1$ the $\mathrm{TE}_{1}$ mode. We will choose $V_{1}, V_{2}$, and $a$ in order to limit our work to couplers where the $\mathrm{TE}_{1}$ mode exists but the $\mathrm{TE}_{2}$ does not, so that the coupled-mode theory using only the two first modes could be used.

\section{A. Case with Kerr-Like Nonlinear Media $\left(\beta_{k}=0\right)$}

We wish to find the dimensionless equation system. Then by introducing (2) in the nonlinear wave equation and making use of the previously defined normalized parameters and dimensionless variables, we obtain the system

$$
\begin{aligned}
& -j \frac{d A_{0}}{d Z} \frac{N_{0}}{n_{1}} \int_{-\infty}^{\infty} \Psi_{0}^{2} d X+e_{k}\left[A_{0}\left(\left|A_{0}\right|^{2} C_{0}+2\left|A_{1}\right|^{2} C_{2}\right)\right. \\
& +A_{0}^{*} A_{1}^{2} C_{2} e^{\left.j 2\left(b_{0}-b_{1}\right) V_{1}^{2} z_{1}\right]=0} \\
& -j \frac{d A_{1}}{d Z} \frac{N_{1}}{n_{1}} \int_{-\infty}^{\infty} \Psi_{1}^{2} d X+e_{k}\left[A_{1}\left(\left|A_{1}\right|^{2} C_{1}+2\left|A_{0}\right|^{2} C_{2}\right)\right. \\
& \left.+A_{1}^{*} A_{0}^{2} C_{2} e^{j 2\left(b_{1}-b_{0}\right) V_{1}^{2} z_{1}}\right]=0
\end{aligned}
$$

where $C_{0}, C_{1}$, and $C_{2}$ are constants depending on the structure defined as

$$
\begin{aligned}
& C_{0}=\int_{-\infty}^{\infty} \Psi_{0}^{4}(\mathscr{X}) d X \quad C_{1}=\int_{-\infty}^{\infty} \Psi_{1}^{4}(X) d X \\
& C_{2}=\int_{-\infty}^{\infty} \Psi_{0}^{2}(X) \Psi_{1}^{2}(X) d X
\end{aligned}
$$

$\mathcal{Z}=z / 2 K_{0} n_{1} d^{2}$ is the dimensionless propagation coordinate and $e_{k}$

$$
e_{k}=k_{o}^{2} d^{2} \alpha_{k} E_{c}^{2} \text {. }
$$

The meaning of $e_{k}$ depends on the definition of the constant $E_{c}$. Following a previous work for the case of a threelayer waveguide [26], first, we will analyze the power of each mode. This can be written making use of the dimensionless parameters in terms of the Poynting vector, such 
as

$$
P_{i}(\mathcal{Z})=\frac{N_{i} c \epsilon_{o} d}{2}\left|A_{i}(\mathcal{Z})\right|^{2} E_{c}^{2} \int_{-\infty}^{\infty} \Psi_{i}^{2}(X) d X
$$

$P_{i}(\mathcal{Z})$, with $i=0,1$ being the mode power, so $P=P_{0}(\mathcal{Z})$ $+P_{1}(Z)$ is the total power, $c$ the speed of light in the free space, and $\epsilon_{o}$ the dielectric permittivity of vacuum. In order to have the coupled-mode system (9)-(10) in terms of normalized parameters, the integral, the effective index, and the refractive index $n_{1}$ that appear in it can be eliminated by imposing

$$
\int_{-\infty}^{\infty} \Psi_{i}^{2}(\mathscr{X}) d X=\frac{n_{1}}{N_{i}}=\frac{1}{\sqrt{1+\left(\delta_{n}^{2}-1\right) b_{i}}}
$$

where a new normalized parameter, $\delta_{n}=n_{2} / n_{1}$ appears. From that we obtain the definition of $E_{c}$ [26] by introducing (13) in (12) as

$$
E_{c}^{2} \equiv \frac{2 P}{n_{1} \epsilon_{o} c d}
$$

Then, if we introduce $E_{c}$ in (11) we can see that $e_{k}$ depends linearly on the total amount of power $P$, and on the nonlinear coefficient $\alpha_{k}$, and it is independent of the other parameters, $e_{k}$ being our power measure. With this definition of $E_{c},(12)$ is written in the simple form

$$
\frac{P_{i}(\mathcal{Z})}{P}=\left|A_{i}(\mathcal{Z})\right|^{2}
$$

the power of each mode being directly associated to the amplitude throughout the propagation. Finally, we rewrite it as $A_{i}=\sqrt{P_{i} / P} e^{j \phi_{i}}$ and we take the following dimensionless variables: $U(\mathcal{Z})=\left[P_{0}(z)-P_{1}(\mathcal{Z})\right] / P$ (the difference between the ratio of power in each mode) and $\theta(\mathcal{Z})=\left(b_{0}-b_{1}\right) V_{1}^{2} \mathcal{Z}-\left[\phi_{0}(\mathcal{Z})-\phi_{1}(\mathcal{Z})\right]$ (the relative phase shift). Then, by introducing these new variables in (9) and (10) and after some mathematical operations the equations become

$$
\begin{aligned}
\frac{d U}{d Z}= & e_{k} C_{2}\left(1-U^{2}\right) \sin 2 \theta \\
\frac{d \theta}{d Z}= & \left(b_{0}-b_{1}\right) V_{1}^{2}+e_{k}\left(\frac{C_{0}-C_{1}}{2}+U \frac{C_{0}+C_{1}}{2}\right. \\
& \left.-U C_{2}(2+\cos 2 \theta)\right)
\end{aligned}
$$

which can be solved in terms of Jacobi elliptic functions [3].

\section{B. The Case with Saturation $\left(\beta_{k} \neq 0\right)$}

In order to simulate the saturation we use the dependence of the refractive index on the electric field given by (1). Now, following the formalism in the same way as in the Kerr-like case and introducing the saturation parameter

$$
s_{k}=\frac{\beta_{k}}{\alpha_{k}^{2}}\left(n_{2}^{2}-n_{1}^{2}\right)
$$

we find that the resulting system is the same as in the previous case, adding a new term due to the saturation, which takes the form

$$
\begin{aligned}
\frac{d U}{d Z}= & U_{k}-e_{k}^{2} \frac{s_{k}}{V_{1}^{2}}\left[\left(1-U-U^{2}+U^{3}\right) Q_{2}\right. \\
& \left.+\left(1+U-U^{2}-U^{3}\right) Q_{1}\right] \sin (2 \theta) \\
\frac{d \theta}{d Z}= & \theta_{k}-e_{k}^{2} \frac{s_{k}}{V_{1}^{2}}\left[G_{0}+G_{1} U+G_{2} U^{2}\right. \\
& +\left(\frac{Q_{1}-Q_{2}}{2}-\left(Q_{2}+Q_{1}\right) U\right. \\
& \left.\left.+\frac{3}{2}\left(Q_{2}-Q_{1}\right) U^{2}\right) \cos (2 \theta)\right]
\end{aligned}
$$

$U_{k}$ and $\theta_{k}$ being the right-hand-side terms in (16) and (17), respectively, $Q_{0}, Q_{1}, Q_{2}$, and $Q_{3}$, being constants defined as

$Q_{0}=\int_{-\infty}^{\infty} \Psi_{0}^{6}(X) d X \quad Q_{1}=\int_{-\infty}^{\infty} \Psi_{0}^{4}(X) \Psi_{1}^{2} X d X$ $Q_{2}=\int_{-\infty}^{\infty} \Psi_{0}^{2}(X) \Psi_{1}^{4}(X) d X \quad Q_{3}=\int_{-\infty}^{\infty} \Psi_{1}^{6}(X) d X$ and $G_{0}, G_{1}$, and $G_{2}$ as

$$
\begin{aligned}
& G_{0}=\frac{\left(Q_{0}+3 Q_{1}-3 Q_{2}-Q_{3}\right)}{4} \\
& G_{1}=\frac{\left(Q_{0}-3 Q_{1}-3 Q_{2}+Q_{3}\right)}{2} \\
& G_{2}=\frac{\left(Q_{0}-9 Q_{1}+9 Q_{2}-Q_{3}\right)}{4} .
\end{aligned}
$$

We are unable to find an analytical solution to this system.

\section{The Hamiltonian}

The Hamiltonian is a suitable tool for obtaining the maximum information when an analytical solution does not exist or is not known. The Hamiltonian satisfies

$$
\frac{\partial H}{\partial \theta}=-\frac{d U}{d Z} \quad \frac{\partial H}{\partial U}=\frac{d \theta}{d Z}
$$

and from here it can be written as

$$
\begin{aligned}
H= & \left(b_{o}-b_{1}\right) V_{1}^{2} U+e_{k}\left[\frac{C_{0}-C_{1}}{2} U\right. \\
& \left.+\left(\frac{C_{0}+C_{1}}{4}-C_{2}\right) U^{2}+\frac{C_{2}}{2}\left(1-U^{2}\right) \cos 2 \theta\right] \\
& -e_{k}^{2} \frac{s_{k}}{V_{1}^{2}}\left[G_{0} U+\frac{1}{2} G_{1} U^{2}+\frac{1}{3} G_{2} U^{3}\right. \\
& +\left(\frac{1}{2} Q_{2}\left(1-U-U^{2}+U^{3}\right)\right. \\
& \left.\left.+\frac{1}{2} Q_{1}\left(1+U-U^{2}-U^{3}\right)\right) \cos (2 \theta)\right]
\end{aligned}
$$


The Hamiltonian and the power parameter $e_{k}$ are our two constants of motion, so if we excite with a given $e_{k}$ and initial $U_{0}$ and $\theta_{0}$, the system will evolve toward these closed points $(U, \theta)$ in order to keep the Hamiltonian constant. This evolution can be plotted in the phase space $(U$, $\theta)$ for each power obtaining the trajectories of motion that will give the information about its behavior.

\section{Theory Limits}

The use of the coupled-mode theory in the analysis of the NLDC provides us with useful insights into its qualitative behavior only if $\Delta n_{N L} / \Delta n_{L}<1$ is verified [1]. In our case $\Delta n_{N L}=\alpha_{k}|E|^{2}-\beta_{k}|E|^{4}$ is the nonlinear index shift and $\Delta n_{L}$ is the smallest difference between linear refractive indexes in the coupler. We have taken into account the condition $\Delta n_{N L} / \Delta n_{L}<0.1$ in order to know whether numerical results are quantitatively or qualitatively correct.

\section{Coupler Behavior with KerR-Like NONLINEARITY}

Below, we analyze the behavior of an NLDC in Kerrlike media using plots of the trajectories of the motion in the phase space. Phase space portraits were first used by Daino et al. in order to explain the instability [13] and more recently to analyze mismatched nonlinear couplers with saturable media [21] using the modes of the individual waveguides. In these works the trajectories in the phase space were plotted for all of the initial excitations at a fixed power in terms of the Stokes parameters. We preferred to plot the trajectories for an initial excitation point and different power values in order to show the behavior in terms of the initial power. In order to understand the meaning of the trajectories in terms of the power traveling within each waveguide, we show in Fig. 2 the fraction of power in a branch in terms of the variable $U$ and $\theta$. So, if we superimpose this figure on the other phase space plots, we can see the power evolution in one branch.

Fig. 3 shows the behavior of a coupler when it is only excited in a branch $\left(U_{0}=0, \theta_{0}=0\right)$. The trajectories for different values of the power parameter $e_{k}$ have been plotted. In this figure we can see the quasi-linear behavior for low power, where the trajectories evolve from the initial phase shift $\theta_{0}=0$ to $\theta=\pi$ after a distance equal to the coupling length, interchanging all of the power between the branches, as can be observed if we superimpose Fig. 2 on Fig. 3. However, note that coupling between modes

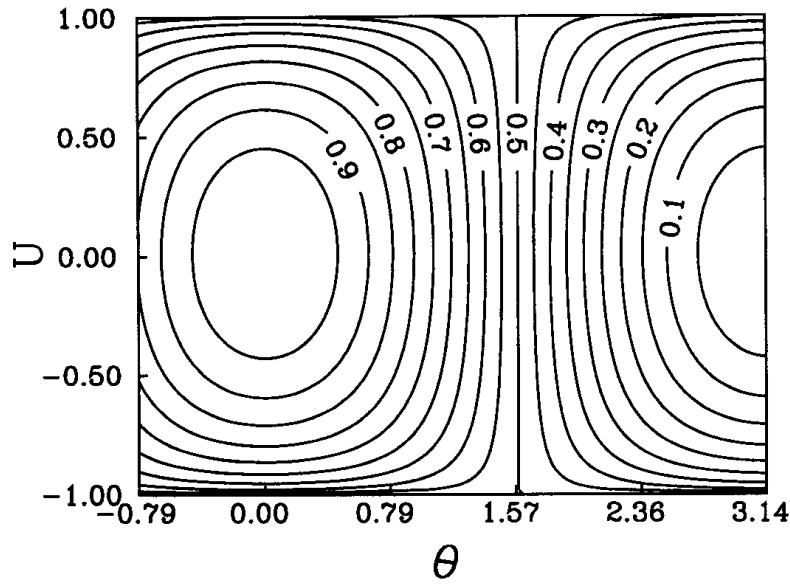

Fig. 2. Lines represent the locus in the phase space with the same relative power distribution. Labels show the fraction of power in one of the coupler branches.

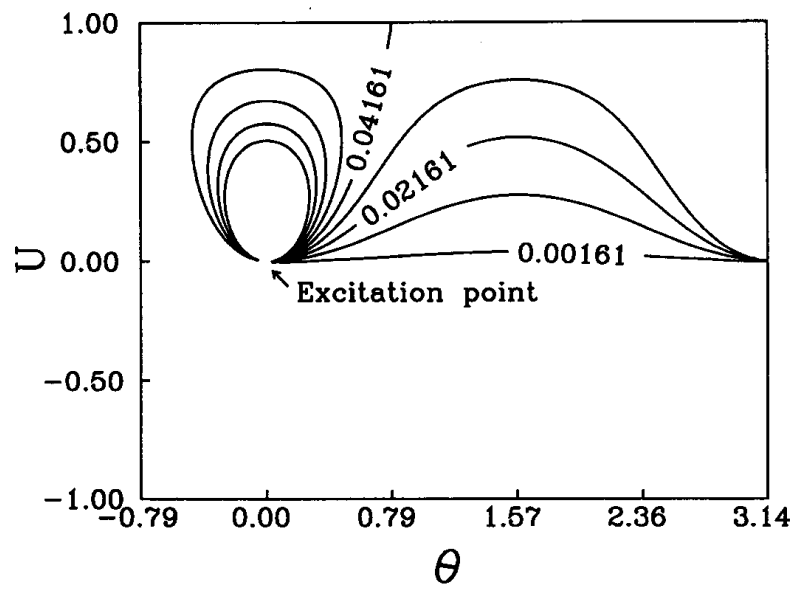

Fig. 3. Trajectories in the phase space for different power values $e_{k}$ when only one branch of the coupler has initially been excited. Trajectory with $e_{k c} \simeq 0.0416$ is the separatrix.

branches is broken. For this power value, the trajectory called the separatrix ends in the symmetric mode ( $U=$ 1), splitting the power between the two waveguides. This value is called the critical power. If the trajectory is made to evolve toward $U=1$ [6], the Hamiltonian can be used to find an analytical expression for the critical power in terms of the normalized parameters as

$$
e_{k c}=\frac{2\left(b_{0}-b_{1}\right) V_{1}^{2}}{\left(1+U_{0}\right)\left[C_{2}\left(2+\cos 2 \theta_{0}\right)-\frac{1}{2}\left(C_{0}+C_{1}\right)\right]+\left(C_{1}-C_{0}\right)}
$$

is null in this case. As the input power increases, there is a growing power exchange between modes until it reaches a value where this situation of total transference between where $U_{0}, \theta_{0}$ correspond to the initial excitation. Above this value the power transfer decreases, the highest exchange being when $U$ is maximum at $\theta=0$ in each pe- 


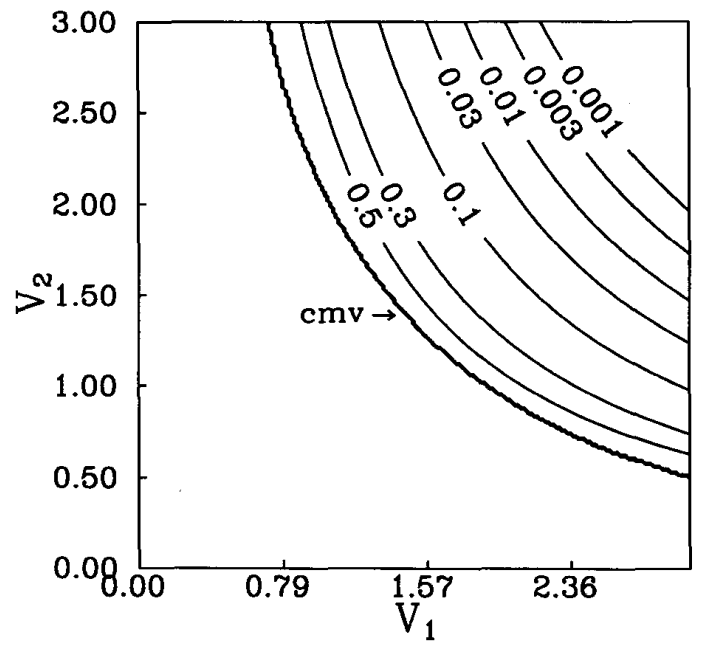

Fig. 4. Locus on the $V_{1}-V_{2}$ plane with the same value of critical power in the case where only one branch of the device has been excited (excitation point $U_{0}=0$ and $\theta_{0}=0$ ). The asymmetric coefficient is $a=0$.

riod, and is always less than one-half the total power. Fig. 4 shows the locus with the identical critical power as a function of the parameters $V_{1}$ and $V_{2}$ (our coupler configuration space) for $a=0$ in the region where only the first two modes exist and taking into account the validity limit of the coupled-mode theory (line labeled $c m v$ ). It could be seen that the region of useful coupler configurations would be smaller if $a>0$ due to the more severe limit of the coupled-mode theory and to the existence of the mode $\mathrm{TE}_{2}$. Also, the critical power values would be higher than in the case with $a=0$.

In Fig. 5 we have plotted the motion trajectories for an initial excitation $U_{0}=-0.5$ and $\theta_{0}=0$. The figure also shows the existence of both subcritical and supercritical working regimes, with the separatrix between them. With this kind of excitation, in the subcritical regime the power is never totally confined in a unique branch and the maximum power is alternatively in one branch or the other. Above the critical power, the maximum is always in the branch that has been excited with the highest power. In this case, only a small amount of power flows between waveguides.

The trajectories for which the coupler is initially excited with a phase shift $\theta_{0}=\pi / 4$ and $U_{0}=0$ are plotted in Fig. 6. Note that in this case the separatrix is almost the same as in Fig. 5. This happens because the behavior is identical when we excite anywhere on one trajectory with the same power parameter $e_{k}$. Here, in the linear regime $\left(e_{k}=0\right)$ the power is totally transferred between the branches, as in the case shown in Fig. 3, but as the input power increases, the fraction of the power that always remains in each branch also increases. Above the critical power only a small amount of power is exchanged from the branch that carries more power to the other, as in the previous case.

Fig. 7 shows the behavior when the coupler is excited

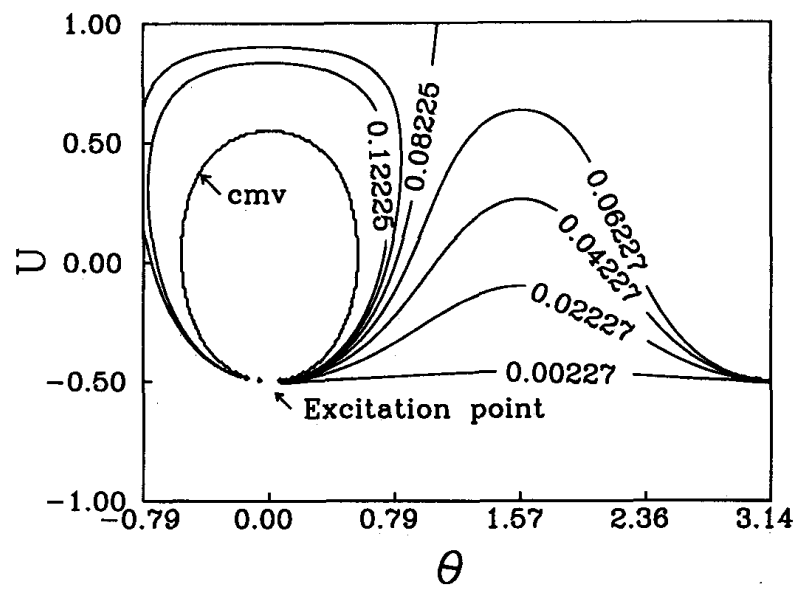

Fig. 5. Phase space portrait when the coupler has been excited in $U_{0}=$ -0.5 and $\theta_{0}=0$. In this case, $e_{k c} \simeq 0.08225$. The line labeled $c m v$ is the validity limit of the coupled-mode theory.

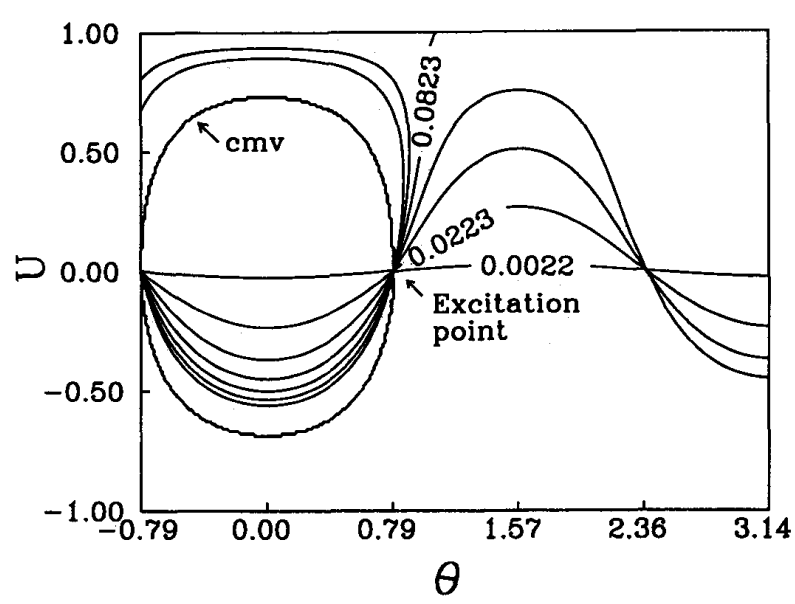

Fig. 6. Trajectories for the excitation point $U_{0}=0$ and $\theta_{0}=\pi / 4$.

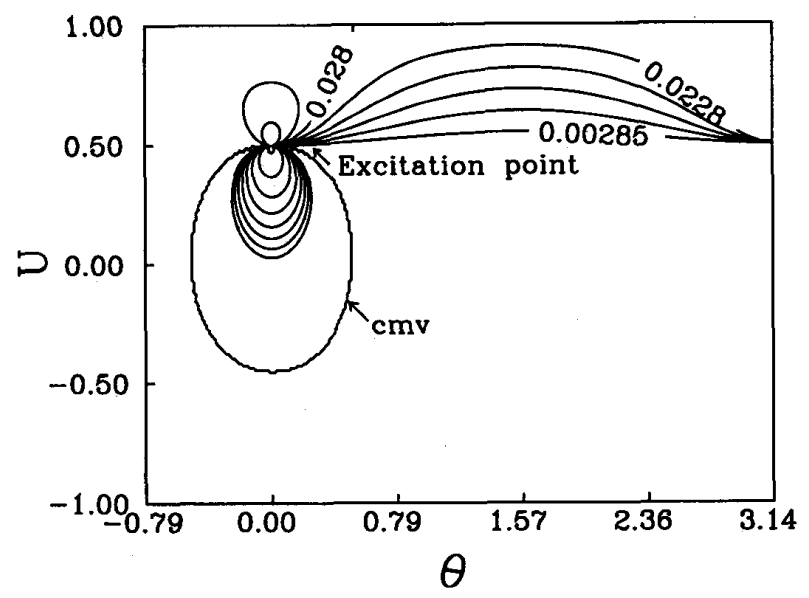

Fig. 7. Trajectories in the phase space for the initial conditions $U_{0}=0.5$ and $\theta_{0}=0$. The critical power value is $e_{k c}=0.0278$ and the fixed point value is $e_{k f} \simeq 0.0425$. 
with $U_{0}>0$ and $\theta_{0}=0$. There is no initial total power transfer between waveguides, the nonlinearity being shown in the $U$ variations until the critical power is reached. It is above this point that we find differences from the previous cases. For a given $U_{0}$, a power value exists whose intensity distribution does not change in the propagation throughout the coupler, $U$ and $\theta$ remaining constant. The coupler behaves as two uninfluenced waveguides or as if the two modes were degenerated. In the local analysis of differential equation systems this value is called a stable fixed point. This value can be easily found from (16) and (17) as

$$
e_{k f}=\frac{2\left(b_{0}-b_{1}\right) V_{1}^{2}}{\left(6 C_{2}-C_{0}-C_{1}\right) U_{0}+C_{1}-C_{0}}
$$

In the supercritical working regime and below this point, the power flows from the waveguide that initially carries the most power to the other during the first half-period. Above this value the opposite occurs, the power being exchanged from the branch that carries less power to the other and for a given power value, all of the power launched in the less excited waveguide is transferred to the other (the trajectory passes through the point $U=0$ and $\theta=0$ ) after a half-period length. In fact, this trajectory can be found when we excite with a $U_{0}>0$ and any $\theta_{0}$.

In Fig. 8 separatrices for different excitations are plotted. Because the behavior of the coupler under any excitation condition is easily deduced when the separatrix is known, this figure gives useful insight into the coupler for all of the excitations. On the other hand, it is also possible to find a critical power whose trajectory evolves toward the antisymmetric mode when we excite near $\theta_{0}=\pi / 2$. However, this trajectory exists for high-power values that are generally beyond the limit of the coupled-mode theory, and we need large separations between waveguides for the trajectory to be considered realistic. The analytical expression that gives this power value can be found from the Hamiltonian as

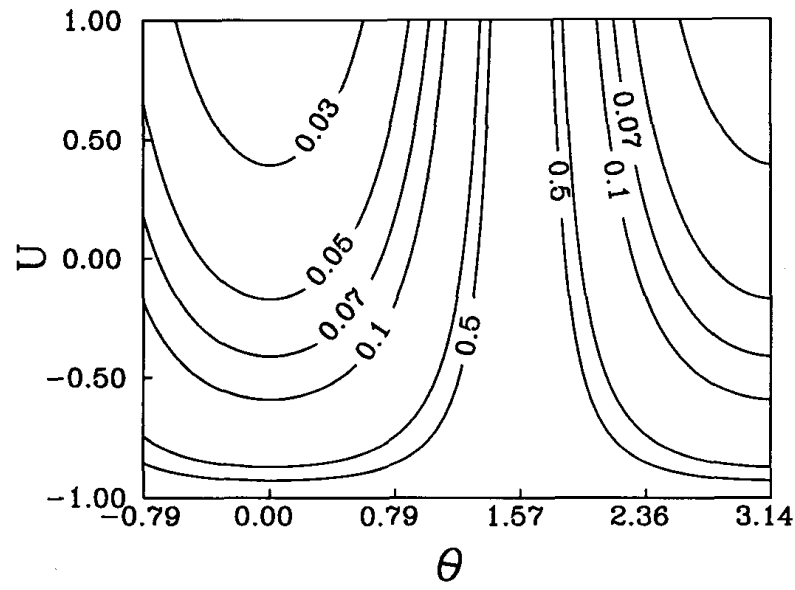

Fig. 8. Separatrices for different excitation points. They can also be understood as the trajectories when we excite in $U_{0}=1$. Note that in this case there is no separatrix for $e_{k}<0.02$.

\section{NLDC BehaVIOR WITH SATURATION $\left(s_{k} \neq 0\right)$}

In order to simulate the saturation of the refractive index, we will take an arbitrary value of $s_{k}=4$, so that $\Delta n_{N L}<0.1 \Delta n_{L}$. Also, we will take as a basis for our study two coupler configurations whose only difference is the normalized separation between waveguides. The first, coupler $A$, is the same as that considered in the previous section, and for the second, coupler $B$, we take $V_{2}=1$.

The behavior of coupler $A$ is basically identical to the Kerr-like case (Fig. 3), but a power increase for similar trajectories is observed. On the contrary, because the nonlinear index shift is not large enough in coupler $B$, the supercritical behavior is not reached, and nonlinearity is only shown in the power exchange between modes, the maximum exchange being plotted by the thick and broken line, as can be seen in Fig. 9. In these cases the critical power obeys the following relation:

$$
e_{k a}=\frac{2\left(b_{0}-b_{1}\right) V_{1}^{2}}{\left(C_{1}-C_{0}\right)-\left(1-U_{0}\right)\left[C_{2}\left(2+\cos 2 \theta_{0}\right)-\frac{1}{2}\left(C_{0}+C_{1}\right)\right]}
$$

So far, all of the figures were drawn for a coupler whose normalized parameters are $V_{1}=2, V_{2}=2, \delta_{n}=1.05$, and $a=0$. A change in the structure, that is, in these parameters, only causes a variation in the value of $e_{k}$ for each trajectory. Therefore, all of the figures can be considered universal plots for each excitation. In fact, when $V_{1}$ or $V_{2}$ decreases or $a$ increases, $e_{k}$ increases for all of the trajectories, as can be seen in Fig. 4 for the particular case of the separatrix. Nevertheless, the changes are irrelevant for the variations in the parameter $\delta_{n}$, as can be seen from the numerical results, because its value must be close to the unit and large variations are not allowed.

$$
\begin{aligned}
0= & \left(b_{0}-b_{1}\right) V_{1}^{2}+e_{k c}\left[\frac{C_{0}-C_{1}}{2}+\left[\frac{C_{0}+C_{1}}{4}\right.\right. \\
& \left.\left.-C_{2}\left(1+\frac{1}{2} \cos \left(2 \theta_{0}\right)\right)\right]\left(1+U_{0}\right)\right] \\
& -e_{k c}^{2} \frac{s_{k}}{V_{1}^{2}}\left[G_{0}+\frac{1}{2} G_{1}\left(1+U_{0}\right)+\frac{1}{3} G_{2}\left(1+U_{0}+U_{0}^{2}\right)\right. \\
& \left.-\frac{1}{2}\left(\left(1-U_{0}^{2}\right) Q_{2}+\left(1+2 U_{0}+U_{0}^{2}\right) Q_{1}\right) \cos \left(2 \theta_{0}\right)\right]
\end{aligned}
$$




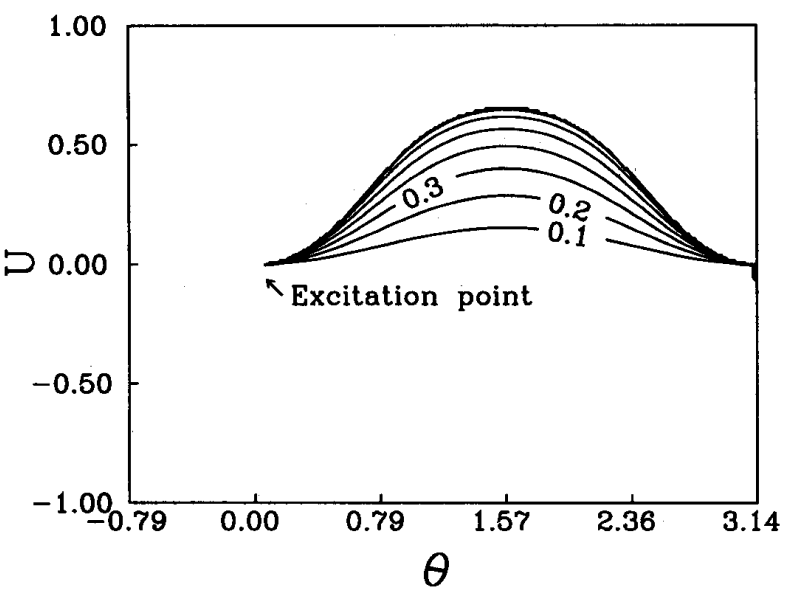

Fig. 9. Trajectories when the coupler is excited in $U_{0}=0$ and $\theta_{0}=0$ for the coupler $B\left(V_{2}=1\right)$.

The saturation of the refractive index requires higher power values to reach the supercritical working regime than in the case without saturation, and it also limits the nonlinear media effects at high power, not allowing the existence of the supercritical behavior or of the stable fixed point $e_{k f}$, depending on the coupler configuration, on the parameter $s_{k}$ and on the initial excitation. Because (26) is a quadratic equation with respect to $e_{k c}$, the solution will exist if its discriminant is positive. This is the condition of existence of the separatrix in terms of the saturation $s_{k}$, the coupler configuration, and the initial excitation. If the separatrices for different excitations of coupler $A$ were plotted (similar to Fig. 8), there would be a zone near $\theta$ $=\pi / 2$ where there is no trajectory, and for coupler $B$ they would only exist for excitations with $U>0.5$ and $\theta<$ $\pi / 4$. For the last coupler, Fig. 10 shows the maximum value $s_{k}$ that the media should have in order to permit the existence of the separatrix for the different possible excitations. Otherwise, for a material with a given value of $s_{k}$, the excitation points where it is theoretically possible to obtain the supercritical behavior are those where the $s_{k}$ of the media is smaller than that in the figure. The saturation would also modify Fig. 4 , so that the zone of coupler configurations where separatrices exist would be smaller, and the limit would not be due to the limit of the coupled-mode theory but to the value of the saturation parameter $s_{k}$. Also, the critical power would be a bit higher than in the case without saturation for each coupler configuration, especially for low values of $V_{1}$.

The power value where there is no intensity change in propagation throughout the coupler (the stable fixed point) can be obtained from (20) as the solution of the quadratic equation

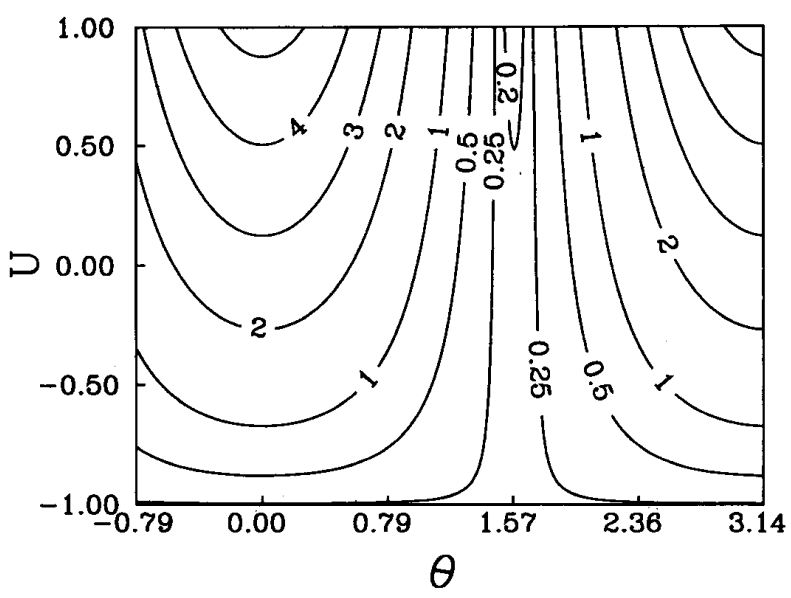

Fig. 10. Locus in the phase space of the maximum saturation parameter $s_{k}$ that allows the existence of the separatrix for the coupler $B\left(V_{2}=1\right)$.

where, as in the case of the critical power, the condition of existence of this effect is that the descriminant must be positive.

As the input power increases, the term $\beta_{k}|E|^{4}$ becomes more important and the coupler returns from the supercritical to the subcritical regime. Here, we also find another separatrix and another stable fixed point. The values for these power inputs are the second solutions of (26) and (27), respectively.

\section{Stability}

Numerical results with the BPM show that the symmetric mode is unstable when launched into the five-layer structure. Previous works gave an explanation of this as a consequence of the bifurcation of motion trajectories given by the evolution of the Stokes parameters in the phase space [13]. In our analysis, the instability appears as a natural consequence of the existence of the separatrix.

In the Kerr-like case, if we pay attention to Fig. 8, where the separatrices for different excitations have been plotted, it is possible to assume another interpretation: the figure can be considered the set of trajectories followed when we excite in $U_{0}=1$. The left-hand-side separatrices start at $-\theta_{l}$ and $U=1$ and end at $\theta_{l}$ and $U=1, \theta_{l}$ depending on the power, and the right-hand-side separatrix starts at $\theta_{r}=\pi-\theta_{l}$ and ends at $\pi+\theta_{l}$. Both of these trajectories are heteroclinic and the points where they start or end are saddle points. This means that the rate of change in $\theta$ and $U$ decreases progressively near the ending point, reaching it for $\mathcal{Z} \rightarrow \infty$. The same happens if this

$$
\begin{aligned}
0= & \left(b_{0}-b_{1}\right) V_{1}^{2}+e_{k f}\left[\frac{C_{0}-C_{1}}{2}+U_{0}\left(\frac{C_{0}+C_{1}}{2}-3 C_{2}\right)\right] \\
& +e_{k f}^{2} \frac{s_{k}}{V_{1}^{2}}\left[G_{0}+\frac{1}{2}\left(Q_{1}-Q_{2}\right)+\left(G_{1}-Q_{1}-Q_{2}\right) U_{0}+\left[G_{2}+\frac{3}{2}\left(Q_{2}-Q_{1}\right)\right] U_{0}^{2}\right]
\end{aligned}
$$


point is excited; a distance $\mathbb{Z} \rightarrow \infty$ is needed to leave it. On the other hand, $U=1$ can be considered a single point, so if we excite the symmetric mode, it will remain at $U$ $=1$. Nevertheless, any small perturbation can cause a change in the motion, therefore a new trajectory close to the separatrix with a higher rate of change could be followed, diverging from the original point and leading to a large variation in the $U$ variable, this being the cause of the first-mode instability. From the above considerations, it is clear that the first-mode instability is due to the existence of the separatrix. If we pay attention to Fig. 8, there are no separatrices with values lower than $e_{k}=0.02$ for our coupler, so if we excite under this value, the symmetric mode will be stable. The exact power value can be found from (23), taking the limit $U \rightarrow 1$ and $\theta \rightarrow 0$, as

$$
e_{k s}=\frac{\left(b_{0}-b_{1}\right) V_{1}^{2}}{3 C_{2}-C_{0}}
$$

which only depends on the coupler parameters.

The cause of instability for the non-Kerr-like nonlinearity case $\left(s_{k} \neq 0\right)$ is also the existence of the separatrix. As in the previous case, we find an expression that provides the power limits of existence of the separatrix from (26) given by

$$
\begin{aligned}
0= & \left(b_{0}-b_{1}\right) V_{1}^{2}+e_{k s}\left(C_{0}-3 C_{2}\right) \\
& -e_{k s}^{2} \frac{s_{k}}{V_{1}^{2}}\left(Q_{0}-2 Q_{1}+2 Q_{2}\right)
\end{aligned}
$$

which yields two solutions. Only between these two solutions will the mode be unstable. As we explained in the previous section, the existence of the separatrix depends on the excitation point, the saturation parameter, and the coupler configuration. Obviously, for couplers where there is no separatrix for any excitation, the first mode will always be stable. From the last expression the value of the saturation parameter that does not allow a coupler to show supercritical behavior (i.e., (29) does not have a solution) is given by

$$
s_{k m}>\frac{\left(C_{0}-3 C_{2}\right)^{2}}{4\left(b_{0}-b_{1}\right)\left(2 Q_{1}-2 Q_{2}+Q_{0}\right)} .
$$

This will also be the condition of nonusefulness of an NLDC, because switching cannot be achieved for any excitation if the saturation parameter of the media is above $s_{k m}$. This parameter only depends on the coupler parameters.

\section{Vi. Conclusions}

We have reported a method for analyzing the behavior and stability of the NLDC by using the Hamiltonian of the coupled-mode system and normalized parameters that allow us to obtain illustrative plots of the trajectories in the phase space showing the existence of subcritical and supercritical behavior separated by the separatrix. Also, we have shown the existence of a kind of excitation where the intensity distribution does not change throughout its evolution for a given power. This power value and the critical power have been analyzed, and an analytical expression has been found for the Kerr-like and non-Kerrlike cases. Furthermore, the instability of the fundamental mode appears as a normal consequence of the existence of the separatrix. The symmetric mode will be unstable above a power value for Kerr-like media or between two values for non-Kerr-like media that only depend on the coupler parameters. Also, an expression is given for the maximum saturation parameter that allows the existence of the separatrix, that is, the possibility of switching in terms of the coupler parameters. All of the work has been done without the need to solve the coupled-mode equations, and the most important characteristics of the coupler behavior have been obtained by taking into account the applicability limit of the coupled-mode theory.

\section{REFERENCES}

[1] G. I. Stegeman, E. M. Wright, N. Finlayson, R. Zanoni, and C. T. Seaton, "Third order nonlinear integrated optics," J. Lightwave Technol., vol. LT-6, pp. 953-970, 1988; see also G. I. Stegeman and E. M. Wright, "All-optical waveguide switching," Opt. Quantum Electron., vol. 22, pp. 95-122, 1990.

[2] S. M. Jensen, "Nonlinear coherent coupler," IEEE J. Quantum Electron., vol. QE-18, pp. 1580-1583, 1982.

[3] Y. Silberberg and G. I. Stegeman, "Nonlinear coupling of waveguide modes," Appl. Phys. Lett., vol. 50, pp. 801-803, 1987.

[4] Y. Chen, "Solution to full coupled wave equations of nonlinear coupled systems," IEEE J. Quantum Electron., vol. 25, pp. 2149-2153, 1989.

[5] X. J. Meng and N. Okamoto, "Improved coupled-mode theory for nonlinear directional couplers,"' IEEE J. Quantum Electron., vol. 27, pp. 1175-1181, 1991.

[6] F. Dios, X. Nogués, and F. Canal, "Critical power in a symmetric nonlinear directional coupler," Opt. Quantum Electron., vol. 24, pp. 1191-1201, 1992.

[7] E. Caglioti, S. Trillo, S. Wabnitz, B. Diano, and G. I. Stegeman, "Power-dependent switching in a coherent nonlinear directional coupler in the presence of saturation," Appl. Phys. Lett., vol. 51, pp. 293-295, 1987.

[8] G. I. Stegeman, C. T. Seaton, C. N. Ironside, T. Cullen, and A. L. Walker, "Effects of saturation and loss on nonlinear directional couplers," Appl. Phys. Lett., vol. 50, pp. 1035-1037, 1987.

[9] E. Caglioti, S. Trillo, S. Wabnitz, and G. I. Stegeman, "Limitations to all-optical switching using nonlinear couplers in the presence of linear and nonlinear absorption and saturation," J. Opt. Soc. Amer. $B$, vol. 5, pp. 472-482, 1988.

[10] E. M. Wright, D. R. Heatley, G. I. Stegeman, and K. J. Blow, "Variation of switching power with the diffusion length in a nonlinear directional coupler," Opt. Commun., vol. 73, pp. 385-392, 1989.

[11] S. Trillo and S. Wabnitz, "Nonlinear nonreciprocity in a coherent mismatched directional coupler," Appl. Phys. Lett., vol. 49, pp. 752$754,1986$.

[12] R. Schiek, "Time-resolved switching characteristic of the nonlinear directional coupler under considerations of susceptibility dispersion,"' IEEE J. Quantum Electron., vol. 27, pp. 2150-2158, 1991.

[13] B. Daino, G. Gregori, and S. Wabnitz, "Stability analysis of nonlinear coherent coupling," J. Appl. Phys., vol. 58, pp. 4512-4514, 1985.

[14] S. Trillo and S. Wabnitz, "Coupling instability and power induced switching with two-core dual polaritates fiber nonlinear couplers," $J$. Opt. Soc. Amer. B, vol. 5, pp. 483-491, 1988.

[15] L. Thylen, E. M. Wright, G. I. Stegeman, C. T. Seaton, and J. V. Moloney, "Beam propagation method analysis of a nonlinear directional coupler," Opt. Lett., vol. 11, pp. 739-741, 1986.

[16] H. D. Feit and J. A. Fleck, "Three-dimensional analysis of a directional coupler exhibiting a Kerr nonlinearity," IEEE J. Quantum Electron., vol. QE-24, pp. 2081-2086, 1988

[17] P. Li Kam Wa, J. E. Sitch, N. J. Mason, J. S. Roberts, and P. N. 
Robson, "All optical multiple-quantum-well waveguide switch," Electron. Lett., vol. 21, pp. 26-28, 1985.

[18] P. R. Berger, P. K. Bhattacharya, and S. Gupta, "A waveguide directional coupler with a nonlinear coupling medium," IEEE J. Quantum Electron., vol. 27, pp. 788-795, 1991.

[19] J. S. Aitchison, A. H. Kean, C. N. Ironside, A. Villanueve, and G. I. Stegeman, "Ultrafast all-optical switching in $\mathrm{Al}_{0.18} \mathrm{Ga}_{0.82} \mathrm{As}$ directional coupler in $1.55 \mu \mathrm{m}$ spectral region,' Electron. Lett., vol. 27, pp. 1709-1710, 1991.

[20] A. W. Snyder, D. J. Mitchell, L. Poladian, D. R. Rowland, and Y. Chen, "Physics of nonlinear fiber couplers," J. Opt. Soc. Amer. B, vol. 8, pp. 986-992, 1991.

[21] Y. Chen, "Mismatched nonlinear coupler with saturable nonlinearity," J. Opt. Soc. Amer. B, vol. 8, pp. 986-992, 1991.

[22] A. T. Pham and Le N. Binh, "All-optical modulation and switching using nonlinear optical directional couplers," J. Opt. Soc. Amer. B, vol. 8, pp. 1914-1931, 1991 .

[23] D. Mihalache, D. Mazilu, M. Bertolotti, and C. Sibilia, “Exact solution for nonlinear thin-film guided waves in higher-order nonlinear media," J. Opt. Soc. Amer. B, vol. 5, pp. 565-570, 1988.
[24] M. Kogelnik and V. Ramaswamy, "Scaling rules for thin-film optical waveguides," Appl. Opt., vol. 13, pp. 1857-1862, 1974.

[25] J. Recolons, L. Torner, and F. Canal, "Normalized parameters for Y-branch optical waveguides," Opt. Lett., vol. 16, pp. 636-638, 1991.

[26] L. Torner and J. P. Torres, "Similarity rules for nonlinear Kerr-like slab optical waveguides," IEEE J. Quantum Electron., vol. 28, pp. 1571-1581, 1992.

David Artigas, photograph and biography not available at the time of publication.

Federico Dios, photograph and biography not available at the time of publication. 